\title{
USO DE FERRAMENTA BIM PARA AUXILIAR NA ESCOLHA DO SISTEMA CONSTRUTIVO QUE GERA MENOR IMPACTO AMBIENTAL ${ }^{1}$
}

\author{
"USING BIM TO ASSIST IN THE SYSTEM CONSTRUCTIVE CHOICE \\ GENERATING LOWER ENVIRONMENTAL IMPACT"
}

\author{
Micheline Helen Cot Marcos \\ Centro Universitário Curitiba (UNICURITIBA) \\ micheline.helen@gmail.com \\ Erica Yukiko Yoshioka \\ Universidade de São Paulo (FAU-USP) \\ ericayy@usp.br
}

\begin{abstract}
Resumo
Esse artigo tem como objetivo principal utilizar uma ferramenta projetual com tecnologia BIM como forma de obtenção de dados de impactos ambientais, durante o processo de desenvolvimento do projeto, para auxiliar profissionais na tomada de decisões quanto ao sistema construtivo que proporcione menor impacto ambiental. Para esse estudo foram analisados o $\mathrm{CO}_{2}$ incorporado e a energia incorporada nos materiais de construção. Para validação da pesquisa, o método adotado foi o estudo de caso onde foram analisados dois sistemas construtivos: aço leve ("steel frame"') e alvenaria. A partir da revisão da literatura foram extraídos os dados de energia e $\mathrm{CO}_{2}$ incorporado dos principais materiais de construção utilizados nos dois sistemas construtivos. Em seguida esses dados foram inseridos em uma ferramenta BIM, sendo agregados aos materiais de construção relativos aos dois sistemas construtivos. Como resultado obtém-se, juntamente com o projeto executivo completo, dados de impactos ambientais, auxiliando o profissional na escolha dos materiais de construção e sistemas construtivos com menor impacto ambiental. Além disso, realiza-se um estudo comparativo entre os sistemas construtivos adotados.
\end{abstract}

Palavras-chave: BIM. Steel Frame. Impactos Ambientais.

\begin{abstract}
This article aims to use a projective tool with BIM as a way to obtain environmental impact data during the project development process to assist professionals in making decisions about building system that provides lower environmental impact. For this study, we analyzed the CO2 embedded and embodied energy in building materials. To validate the research, the method adopted was the case study which analyzed two building systems: light steel ("steel frame" ') and masonry. From the literature review were extracted energy data and CO2 built the main building materials used in both building systems. Then these data were entered into a BIM tool, being added to construction materials for the two building systems. As a result is obtained, along with the complete executive project, environmental impact data, helping the professional in the choice of building materials and construction systems with lower environmental impact. In addition, there will be a comparative study between construction systems adopted. Tools "Building Information Modeling" (BIM) represent a new generation of object-oriented computational tools that manage the construction of the information in the project life cycle.
\end{abstract}

Keywords: BIM. Steel Frame. Environmental Impacts.

\footnotetext{
${ }_{1}$ MARCOS, M.; YOSHIOKA, E. Uso de ferramenta BIM para auxiliar na escolha do sistema construtivo que gera menor impacto ambiental In: ENCONTRO BRASILEIRO DE TECNOLOGIA DE INFORMAÇÃO E COMUNICAÇÃO NA CONSTRUÇÃO, 7., 2015, Recife. Anais... Porto Alegre: ANTAC, 2015.
} 


\section{IMPACTOS AMBIENTAIS NA CONSTRUÇÃO CIVIL E USO DE BIM}

Novas tecnologias construtivas aliadas a novas tecnologias projetuais contribuem para a melhoria do ambiente construído na área de eficiência energética, desempenho térmico e impactos ambientais (FREIRE, M.; AMORIM, A., 2011). Como descreve Agopyan; John (2011), as decisões de projeto e a especificação de materiais construtivos e seus componentes, afetam diretamente o consumo de recursos naturais de energia. Com relação ao consumo desses recursos, a construção civil está se posicionando e tomando decisões cada vez mais relevantes.

Esse artigo descreve como mensurar impactos ambientais causados pela indústria da construção civil, através do uso de ferramenta com tecnologia BIM ("Building Information Modeling"). O objetivo principal da pesquisa é de auxiliar o profissional do setor de projetos na tomada de decisões quanto ao sistema construtivo que proporcione menor impacto ambiental, no momento de desenvolvimento do projeto.

O uso de ferramentas BIM no processo de projeto do edifício contribui para a melhoria da qualidade das construções, seu desempenho térmico e na escolha dos materiais e sistemas construtivos que propiciam redução de impactos ambientais. $\mathrm{Na}$ fase de projeto, as tecnologias BIM proporcionam ao profissional a possibilidade de conceber um modelo parametrizado, no qual é possível visualizar a volumetria, verificar os impactos da incidência solar, quantificar e qualificar o material aplicado, com ajustes de variáveis de conforto e impactos ambientais (FREIRE; AMORIM, 2011).

A construção civil no Brasil carece de definições quanto aos impactos ambientais causados pelo consumo de energia relacionado às edificações, desde a fabricação, manutenção e consumo dos materiais de construção, o levantamento da obra, até o consumo de energia durante o ciclo de vida. Essa carência de informações e definições é mostrada na pesquisa feita pelo CBCS (Conselho Brasileiro de Construção Sustentável) juntamente com o PNUMA (Programa das Nações Unidas para o Meio Ambiente) (CBCS; PNUMA, 2014), onde mostra que os profissionais da área da construção civil gostariam de ter acesso a mais ferramentas computacionais de simulação energética (28\%). Para a área de materiais, apontam a necessidade de criar um banco de dados público de Análise do Ciclo de Vida com dados de produtos e fabricantes nacionais. Para a área hídrica, há falta de ferramentas para auxiliar na implantação de fontes alternativas de água.

Dados que provam a importância de se pensar em reduzir impactos ambientais ocasionados pela construção civil. A preocupação mundial em fazer construções ambientalmente conscientes com a sua inserção no espaço vem do fato de que os edifícios consomem mais da metade de toda a energia usada nos países desenvolvidos e que produzem mais da metade dos gases poluentes ao meio ambiente (ROAF; FUENTES; THOMAS, 2006). Esse consumo vem desde a extração da matéria prima, passando pela fabricação dos materiais de construção, erguimento da edificação, uso e demolição

Os envolvidos, como são mostrados na Figura 1, sugerem que sejam criados bancos de dados públicos de livre acesso, com parâmetros ambientais como vida útil, conforto térmico, consumo energético, emissão de $\mathrm{CO}_{2}$, dentre outros aspectos. Solicitam a criação de uma base brasileira pública que tenha interface com ferramentas de gestão do tipo "Building Information Modeling" (BIM). 
Figura 1 - Pesquisa realizada pela CBCS e PNUMA sobre a necessidade de dados pertinentes à solicitação de ferramentas específicas para reduzir impactos ambientais relativos à construção civil.

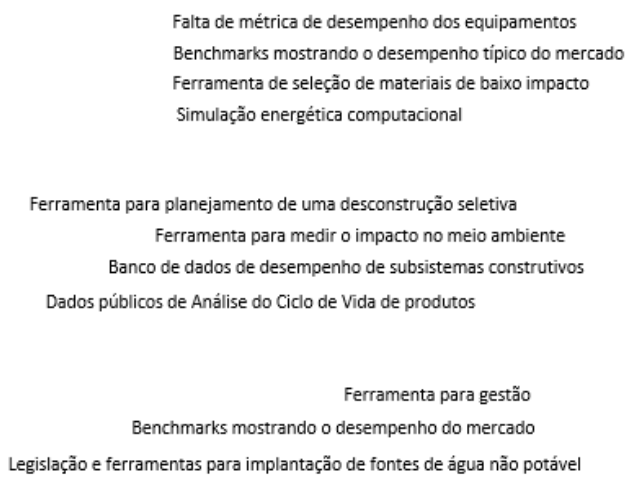

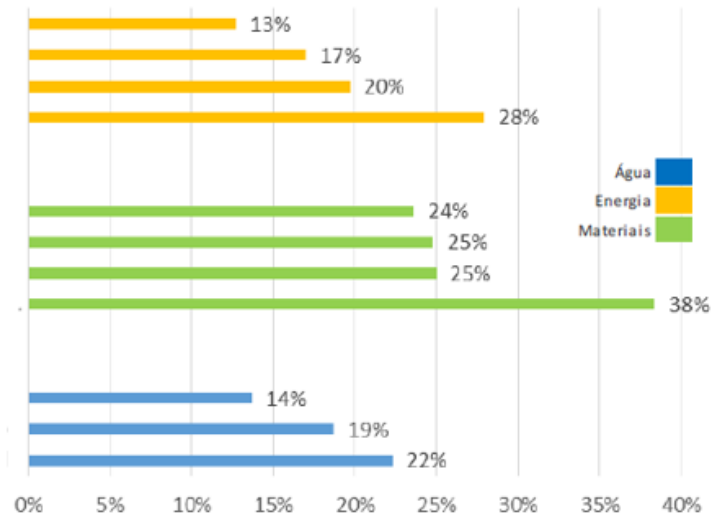

Fonte: CBCS; PNUMA, 2014

As certificações ambientais internacionais, no setor da construção civil no país, ganharam destaque a partir de 2007, principalmente em projetos comerciais e de alto padrão. No ano de 2012, a busca pela certificação de sustentabilidade nas construções representou $9 \%$ do valor da indústria de construção civil (ERNST; YOUNG, 2013). Certificações no Brasil incluem as de origem internacional, como LEED ("Leadership for Energy and Environmental Design") e adaptações brasileiras de certificações de outros países como o AQUA (Alta Qualidade Ambiental). Também foram desenvolvidas certificações setoriais, como o Selo Qualiverde, na cidade do Rio de Janeiro; Selo BH Sustentável em Belo Horizonte e o Selo Casa Azul da Caixa, que é direcionado às construções do Programa Minha Casa Minha Vida, do Governo Federal. As principais certificações ambientais utilizadas no Brasil estão listadas no Quadro 1.

Quadro 1 - Principais certificações ambientais relacionadas à construção civil no Brasil.

\begin{tabular}{|l|l|}
\hline \multicolumn{1}{|c|}{ Certificação } & \multicolumn{1}{c|}{ Abrangência } \\
\hline LEED & Internacional \\
\hline BREEAM & Internacional \\
\hline AQUA & Brasil \\
\hline Selo Casa Azul & Brasil - Programa Minha Casa Minha Vida \\
\hline PBE Edifica (Procel) & Brasil \\
\hline Selo Qualiverde & Brasil - Rio de Janeiro \\
\hline Referencial Casa & Brasil \\
\hline Selo BH Sustentável & Brasil - Belo Horizonte \\
\hline
\end{tabular}

Fonte: CBCS; PNUMA (2014), apud MARCOS (2015)

De acordo com o Ministério do Meio Ambiente (MMA), o setor da construção civil tem papel fundamental para a diminuição dos impactos ambientais. Além dos impactos relacionados aos 
materiais e energia, existem aqueles associados à geração de resíduos sólidos, líquidos e gasosos. Na construção civil os impactos ambientais se refletem em todas as atividades e implicam na revisão dos procedimentos que propiciam um alto consumo de materiais, geração de resíduos, emissão de gases geradores do efeito estufa e no consumo de água e energia (AGOPYAN; JOHN, 2011).

\section{APLICAÇÃO DA FERRAMENTA BIM}

Para aplicação da ferramenta BIM como forma de mensurar impactos ambientais, foram utilizados dois sistemas construtivos, a fim de obter um comparativo de resultados ao final: alvenaria convencional e "steel frame". As análises foram feitas em uma parede projetada no software ArchiCad, detalhada nos dois sistemas construtivos. Com relação aos impactos ambientais, foram analisados o $\mathrm{CO}_{2}$ incorporado e a energia incorporada nos materiais de construção relativos aos dois sistemas construtivos.

O sistema steel frame: A palavra "steel" significa a matéria prima usada na estrutura, o aço. A inclusão de "Light" significa leve, indica que os elementos em aço são de baixo peso uma vez que são produzidos a partir de chapa de aço com espessura reduzida. A palavra "Frame", significa estrutura. Nessa pesquisa foi utilizado o sistema "steel frame" com estrutura em aço leve, então pode-se assim denominar: "light steel frame". O sistema "Light Steel Framing", é um sistema construtivo de concepção racional, que tem como principal característica uma estrutura constituída por perfis formados a frio de aço galvanizado que são utilizados para a composição de painéis estruturais e não-estruturais, vigas secundarias, vigas de piso, tesouras de telhado e demais componentes (Figura 2 e 3). Por ser um sistema industrializado, possibilita uma construção a seco com rapidez de execução, por isso o sistema "Light Steel Framing" pode ser conhecido por Sistema autoportante de construção a seco.

Figura 2 - Estrutura de residência em light steel frame com fechamentos em placa cimentícia. Moradia construída para habitação de interesse social, em Curitiba - Paraná.

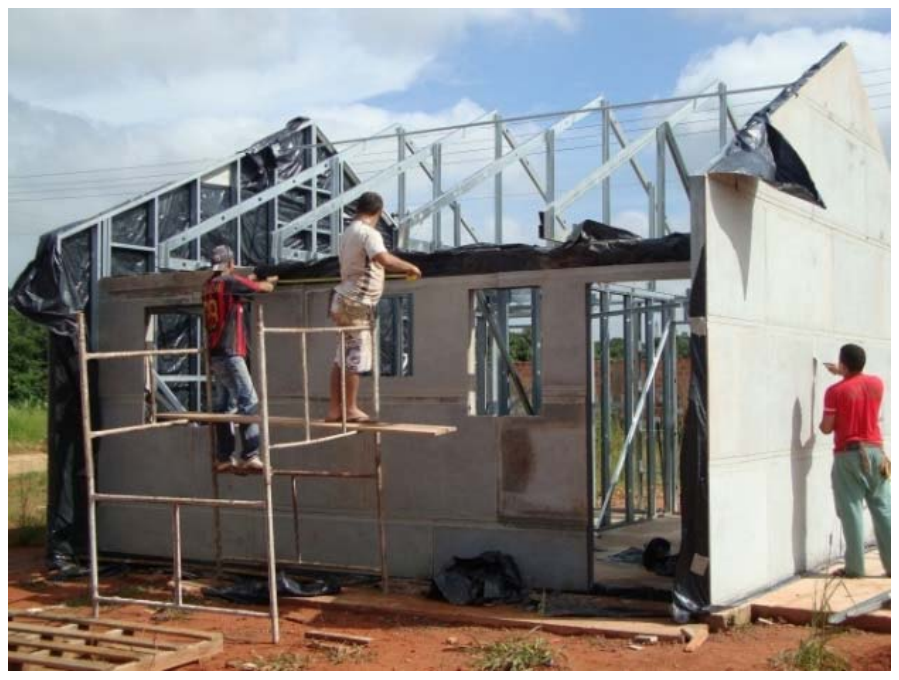

Fonte: A autora (acervo pessoal). 
Figura 3 - Montagem dos perfis de steel frame

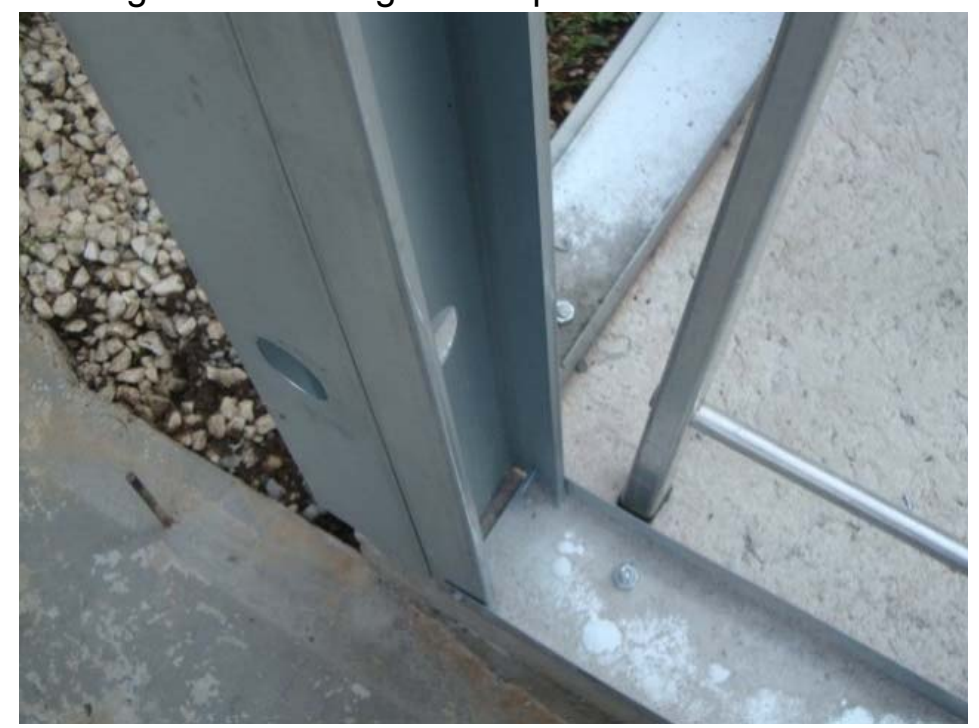

Fonte: A autora (acervo pessoal).

O mercado brasileiro tem mostrado que o uso de novas tecnologias construtivas e projetuais é a melhor forma de permitir a industrialização e a racionalização dos processos. Uma observação importante é que a utilização do aço como sistema construtivo demanda profissionais preparados, projetos detalhados e integrados, como ocorre com as ferramentas BIM. Nesse aspecto o uso do aço na construção civil se torna uma das alternativas para mudar o panorama do setor (CBCA, 2014).

No processo utilizando a tecnologia BIM, os modelos virtuais podem ser entendidos como bases de dados onde são armazenados tanto os dados geométricos, como os textuais de cada elemento construtivo utilizado no projeto. A combinação desses dados permite a extração automática de documentos como plantas, cortes, perspectivas ou quantitativos. A atenção do projetista é, portanto, destinada primordialmente às soluções projetuais e não aos desenhos técnicos, que são em boa parte gerados automaticamente pela ferramenta (BIRX, 2006). As vantagens do uso da modelagem vão muito além da criação de maquetes eletrônicas e agilização do processo de produção de documentações projetuais. Assim como nas indústrias metalomecânica, manufatureira e aeroespacial, a visualização tridimensional do modelo permite verificar as inadequações e incompatibilidades instantaneamente, auxiliando nos processos de decisão de maneira intuitiva, em todas as etapas do projeto.

Outro ponto importante é a consolidação das informações que constituem o projeto. Uma vez que se utiliza uma base de dados unificada para todo o conteúdo de informação, as modificações em um determinado documento (por exemplo, uma planta baixa do projeto arquitetônico), propagam-se para os demais documentos envolvidos automaticamente, garantindo assim a agilidade nas atualizações, modificações e confiabilidade no acesso às informações.

\subsection{Modelagem e análise de dados na ferramenta BIM}

De acordo com Scheer; Ayres (2007), a visualização em três dimensões acrescentada pelo uso de um CAD 3D aumenta a quantidade de informações do projeto. Entretanto, os CADs 3D apresentam a mesma característica de fragmentação da informação dos CADs geométricos, tornando difícil a produção de informações estruturadas, que normalmente constituem o núcleo da documentação de um projeto (plantas, cortes, elevações, etc.).

Por exemplo, ao invés de representar paredes através de linhas paralelas, utiliza-se o 
elemento parede, que além de ser armazenado e interpretado pelo computador como a representação de uma parede, possui um comportamento específico que inclui: se estender apenas longitudinalmente (a extensão transversal é a espessura), possuir determinada altura, a capacidade de receber aberturas (portas e janelas), se associar corretamente a outros elementos parede (eliminando arestas desnecessárias nos encontros de elementos), etc. Além disso, o elemento parede possui informações relativas à sua composição e aparência: material de acabamento, de revestimento, do núcleo; e também informações utilizadas na representação bidimensional do elemento: cor, espessura do traço, hachura, etc. (IBRAHIM et al., 2004).

Para ilustrar o uso dessa ferramenta, encontra-se a seguir, nas Figuras 4 e 5, duas imagens geradas através da ferramenta BIM (ArchiCad). O projeto demonstra um dos modos de visualização e como as especificações são mostradas (MARCOS, 2009).

Figura 4 - Modelo de projeto com modelagem das informações.

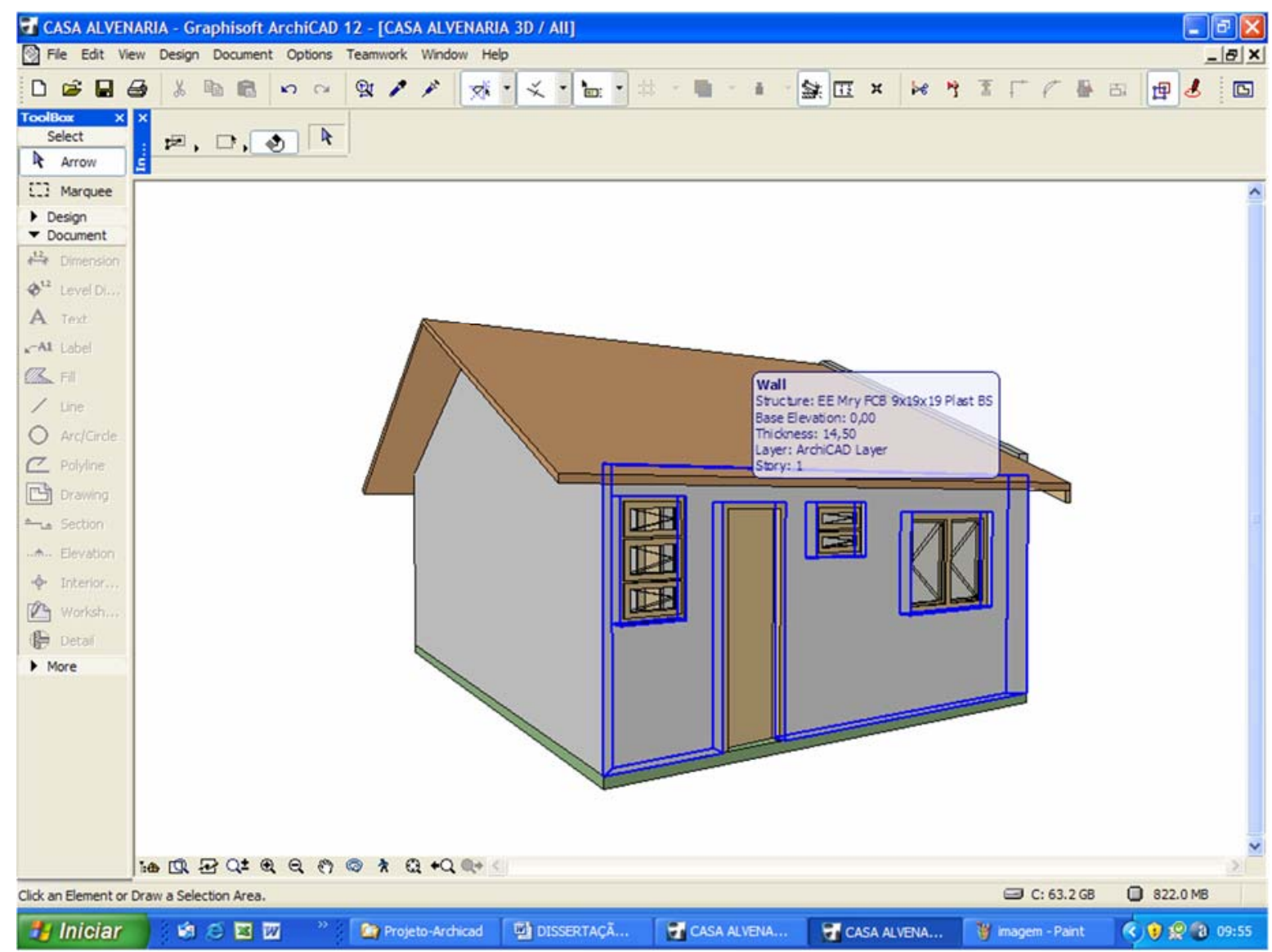

Fonte: MARCOS, M. (2009) 
Figura 5 - Modelo de projeto com modelagem das informações.

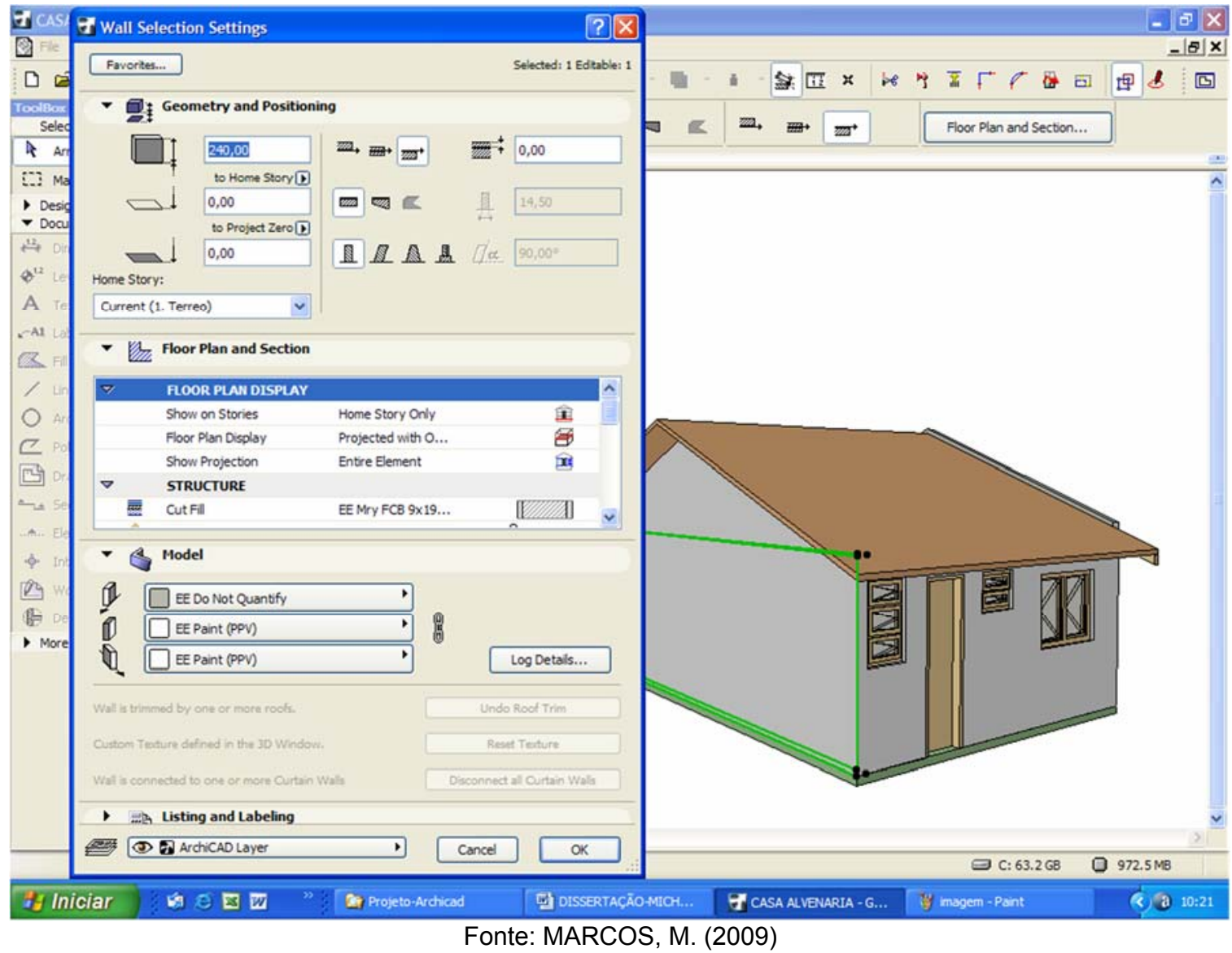

As informações relativas ao que é selecionado são mostradas como, por exemplo, selecionase o elemento parede, então as informações como altura, largura, profundidade, especificação de material, acabamento e outras informações que se pode agregar ao banco de dados, aparecem no visor. No caso dessa pesquisa, informações relativas à energia incorporada e $\mathrm{CO}_{2}$ referente a cada material de construção, são agregadas ao banco de dados. Outros dados podem ser fornecidos à ferramenta e assim, obter mais resultados no processo de projeto.

O uso de ferramentas para avaliar impactos ambientais que agregam informações ao projeto desenvolvido facilita as análises de gastos energéticos, emissão de poluentes ao meio ambiente, entre outros fatores. Com isso, tem-se a possibilidade de atenuar problemas ambientais a partir do projeto, antes mesmo de se iniciar a obra.

Na Figura 6 abaixo está esquematizado o processo de informação do ArchiCAD. Primeiramente o elemento (nesse caso, parede) é desenhado, em seguida aplicam-se os materiais e acabamentos relativos a essa parede. A base de dados está alimentada com ferramentas de associação automáticas que indicam os parâmetros de carbono incorporado, em quilos, gerando uma lista de resultados. 
Figura 6 - Processo de informação na plataforma BIM ( ArchiCad)

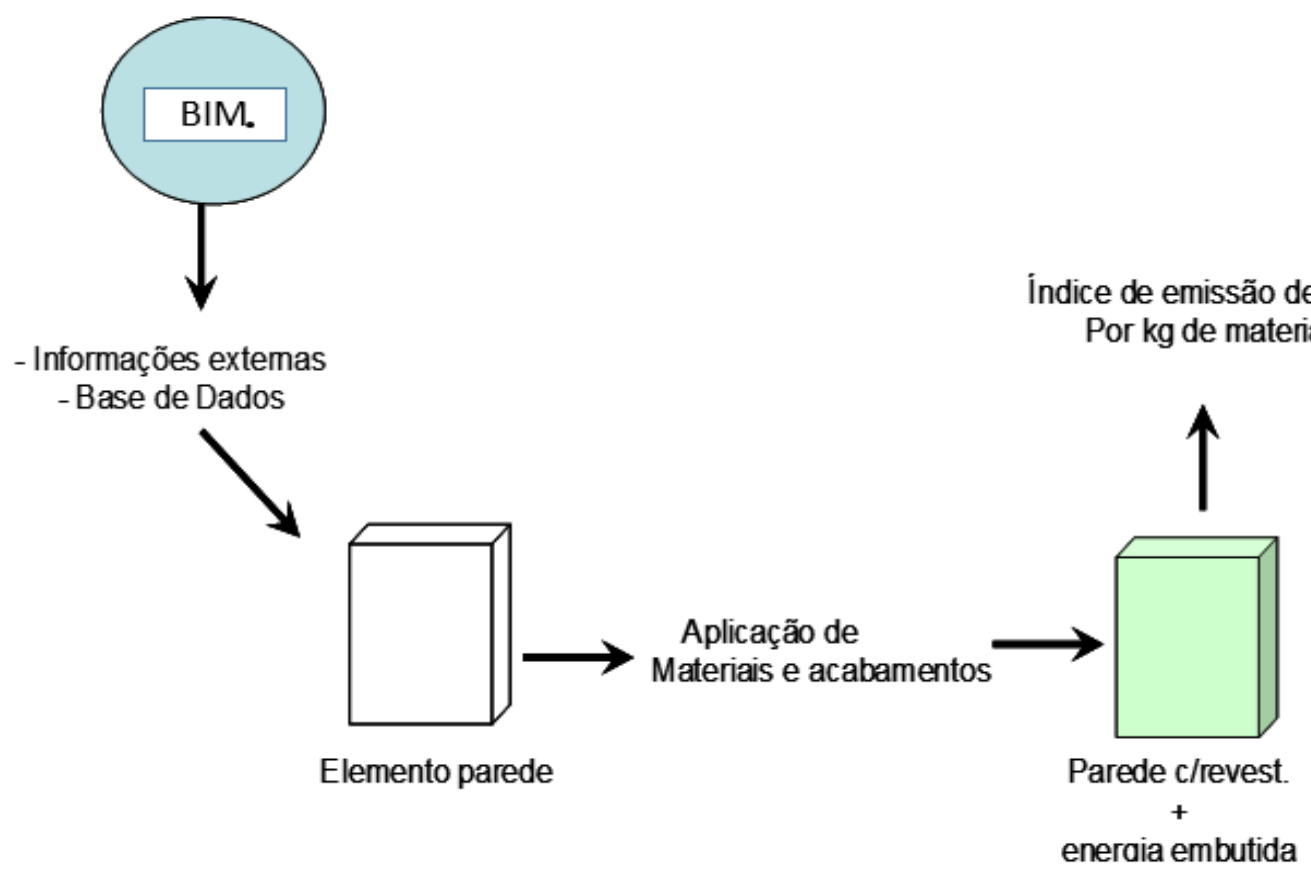

Fonte: MARCOS, M. (2009)

Assim foram obtidos resultados referentes aos dois sistemas construtivos: alvenaria e "steel frame", com relação ao $\mathrm{CO}_{2}$ incorporado e energia incorporada. Com a utilização desse método, é possível obter dados de diversos impactos ambientais e analisá-los no momento da concepção do projeto como forma de auxiliar na escolha do sistema construtivo com menor impacto ambiental.

\section{RESULTADOS E CONCLUSÂO}

A parede analisada nessa pesquisa faz parte da residência, conforme planta baixa e está destacada em azul.

Figura 7- Parade analisada na pesquisa, em destaque.

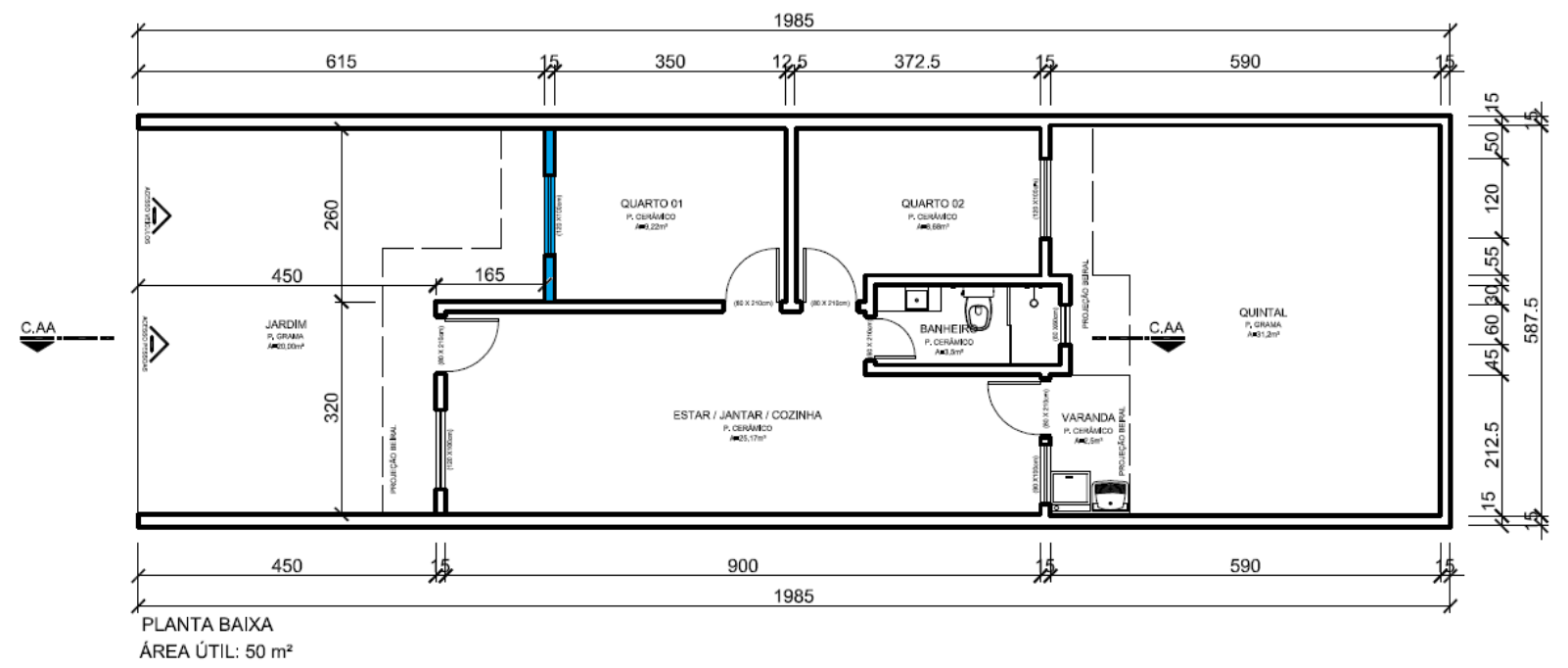

Fonte: MARCOS, M. (2015) 
A seguir, no Quadro 2, têm-se os valores de carbono e energia incorporados para uma parede.

Quadro 2 - Dados extraídos do ArChicad referentes a uma parede em alvenaria.

\begin{tabular}{|l|c|c|}
\hline Material & Carbono Embutido $(\mathbf{K g ~ C O} \mathbf{~})$ & Energia Incorporada (MJ) \\
\hline Chapisco & 16,31 & 215,43 \\
\hline Tijolo $-9 \mathrm{~cm}$ & 319,24 & $1.641,49$ \\
\hline Total & 335,55 & $\mathbf{1 . 8 5 6 , 9 1}$ \\
\hline
\end{tabular}

Fonte: MARCOS, M. (2015)

Quadro 3 - Dados extraídos do ArChicad referentes a uma parede em steel frame.

\begin{tabular}{|l|r|r|}
\hline Material & CO2 Incorporado $\left(\mathbf{K g C O}_{\mathbf{2}}\right)$ & Energia Incorporada (MJ) \\
\hline Aço - Estrutural & 52,97 & $\mathbf{7 4 6 , 1 1}$ \\
\hline Gesso Acartonado & 114 & 838,45 \\
\hline Placa Cimenticia & 96,55 & $1.609,13$ \\
\hline Total & $\mathbf{2 6 3 , 5 2}$ & $\mathbf{3 . 1 9 3 , 6 9}$ \\
\hline
\end{tabular}

Fonte: MARCOS, M. (2015)

Com isso, observa-se que, para a construção de uma parede individual em steel frame, haverá um consumo de $44 \%$ de $\mathrm{CO}_{2}$ incorporado e de $56 \%$ se a mesma parede for construída em alvenaria. E para a construção de uma parede individual em "steel frame" haverá um consumo de $63 \%$ de energia incorporada e de $37 \%$ se a mesma parede for construída em alvenaria.

As análises energéticas, no sistema BIM, podem ser realizadas para todo o empreendimento ou apenas para um único elemento como mostra esse estudo. A utilização dessa metodologia para desenvolvimento de projeto auxilia o profissional na escolha dos materiais de construção e consequentemente dos sistemas construtivos mais eficientes ambientalmente, no momento da realização do projeto. Dessa maneira profissionais da área da construção civil podem contribuir para a redução de impactos ambientais no momento da realização do projeto, podendo assim fazer escolhas de diferentes tipologias construtivas conforme os resultados das análises.

\section{REFERÊNCIAS}

AYRES, C.; SCHEER, S. Diferentes abordagens do uso do CAD no processo de projeto arquitetônico. In: Workshop Brasileiro de gestão do processo de projetos na construção de edifícios, 2007, Curitiba, PR.

BIRX, G. W. Getting started with Building Information Modeling. The American Institute of Architects - Best Practices, 2006. Disponível em http://www.aia.org/bestpractices_index. Acessado em: 02/02/2009

CBCA (Centro Brasileiro de Construção em Aço). Steel Framing: Arquitetura. Série: Manual de Construção em Aço, 2014. Disponível em: http://www.cbca-acobrasil.org.br. Acessado em: 02/2015. 
CBCS (Conselho Brasileiro de Construção Sustentável); PNUMA (Programa das Nações Unidas para o Meio Ambiente). Aspectos da Construção Sustentável no Brasil e Promoção de Políticas Públicas. Ministério do Meio Ambiente, Governo Federal, 2014.

ERNST; YOUNG. Sustainable buildings in Brazil. GBC Brasil: 2013.

FREIRE, M.R.; AMORIM, A.L. A abordagem BIM como contribuição para a eficiência energética no ambiente construído. TIC- 2011. Encontro de Tecnologia de Informação e Comunicação na Construção. Salvador, 2011.

IBRAHIM, M; KRAWCZYK, R; SCHIPPORIET, G. Two Approaches to BIM: A Comparative Study, 2004.Disponível em: http://www.iit.edu/ ibramag/ acessado em fev. 2009

JOHN, V.; AGOPYAN, G. O desafio da Sustentabilidade na Construção Civil. Editora: Blucher. Volume 5. Sáo Paulo, 2011

MARCOS, M. Análise de emissão de $\mathrm{CO} 2$ na fase pré-operacional da construção de habitações de interesse social através da utilização de uma ferramenta CAD-BIM. Dissertação de mestrado apresentada ao programa de Pós Graduação em Construção Civil, UFPR, Curitiba - Paraná, 2009.

MARCOS, M. Método de obtenção de dados de impactos ambientais, durante o processo de desenvolvimento do projeto, através do uso de ferramenta BIM. Tese de Doutorado apresentada ao programa de Pós Graduação em Arquitetura e Urbanismo, FAU-USP, São Paulo - SP, 2015.

PINHEIRO, M., D. Ambiente e Construção Sustentável. Instituto do Ambiente. Portugal, Lisboa, 2006.

ROAF, Sue; FUENTES, Manuel; THOMAS Stephanie. Ecohouse - A Casa Ambientalmente Sustentável. $2^{\mathrm{a}}$ edição. Porto Alegre: Bookman, 2006. 